\title{
Perfil sociodemográfico, ocupacional e avaliação das condições de saúde mental dos trabalhadores da Estratégia Saúde da Família em um município do Rio Grande do Sul, RS
}

\author{
Sociodemographic and occupational profile and evaluation of mental health disorders of \\ employees of the Family Health Strategy in a city of Rio Grande do Sul, RS
}

\section{Perfil sociodemografico, ocupacional y evaluación de las condiciones de salud mental de los trabajadores de Estrategia de Salud de la Familia en un municipio de Rio Grande do Sul, RS}

Izadora Joseane Borrajo Moreira. Universidade de Santa Cruz do Sul (UNISC). Santa Cruz do Sul, RS, Brasil. izadoramoreira@yahoo.com.br (Autora correspondente) Jorge André Horta. Universidade de Santa Cruz do Sul (UNISC). Santa Cruz do Sul, RS, Brasil. jhorta @ unisc.br Luciano Nunes Duro. Universidade de Santa Cruz do Sul (UNISC). Santa Cruz do Sul, RS, Brasil. Iduro2009@gmail.com Daniela Teixeira Borges. Universidade de Santa Cruz do Sul (UNISC). Santa Cruz do Sul, RS, Brasil. med.dani@ hotmail.com Anderson Berni Cristofari. Universidade de Santa Cruz do Sul (UNISC). Santa Cruz do Sul, RS, Brasil. anderson.cristofari@ hotmail.com Jéssica Chaves. Universidade de Santa Cruz do Sul (UNISC). Santa Cruz do Sul, RS, Brasil. jessykasacht@yahoo.com.br Débora Cristina Haack Bassani. Universidade de Santa Cruz do Sul (UNISC). Santa Cruz do Sul, RS, Brasil. deborachb08@gmail.com Emanuelle Damo Cerizolli. Universidade de Santa Cruz do Sul (UNISC). Santa Cruz do Sul, RS, Brasil. edcerizolli@ hotmail.com Raquel Montagna Teixeira. Universidade de Santa Cruz do Sul (UNISC). Santa Cruz do Sul, RS, Brasil. quelmteixeira@gmail.com

\section{Resumo}

Objetivo: Descrever os fatores sociodemográficos e ocupacionais e avaliar a prevalência de transtornos mentais comuns em trabalhadores das Estratégias de Saúde da Família (ESF) em Santa Cruz do Sul, RS. Métodos: Estudo com abordagem epidemiológica, de corte transversal e quantitativo, realizado no período de junho a agosto de 2013. Foram avaliados 83 trabalhadores, entre médicos, enfermeiros, técnicos/auxiliares de enfermagem e agentes comunitários de saúde, atuantes nas 11 unidades de saúde da família do município. O instrumento de pesquisa constituiu-se em um questionário autoaplicável, englobando aspectos sociodemográficos, ocupacionais e questões pertencentes ao Self-Reporting Questionnaire. Resultados: A idade média dos participantes foi de 37,1 $\pm 8,9$ anos, sendo a maioria agentes comunitários de saúde, do sexo feminino, casadas, com filhos, renda per capita de até 2 salários mínimos e ensino médio completo. A prevalência geral de transtorno mental comum foi de 19,7\%, estando presente em todas as categorias profissionais, e mais frequente entre os enfermeiros (25\%), porém sem diferenças estatísticas. Conclusão: O presente estudo, além de caracterizar a população de trabalhadores das ESF de Santa Cruz do Sul, RS, em seus aspectos sociodemográficos e ocupacionais, identificou que o sofrimento psíquico está presente, embora em graus variados, em todas as categorias profissionais avaliadas. Frente a esses achados relevantes, sugerem-se providências que visem à prevenção e promoção da saúde mental desses profissionais.

\section{Palavras-chave:}

Atenção Primária à Saúde

Saúde do Trabalhador

Transtornos Mentais

Estresse Psicológico

Agentes Comunitários de Saúde
Como citar: Moreira IJB, Horta JA, Duro LN, Borges DT, Cristofari AB, Chaves J et al. Perfil sociodemográfico, ocupacional e avaliação das condições de saúde mental dos trabalhadores da Estratégia Saúde da Família em um município do Rio Grande do Sul, RS. Rev Bras Med Fam Comunidade. 2016;11(38):1-12. http://dx.doi.org/10.5712/rbmfc11(38)967
Fonte de financiamento: declaram não haver. Parecer CEP: 161.000 (UNISC), aprovado em 03/12/2012.

Conflito de interesses: declaram não haver. Procedência e revisão por pares: revisado por pares. Recebido em: 06/07/2014. Aprovado em: 06/01/2016. 


\begin{abstract}
Objective: The purpose of this study was to measure the prevalence of common mental health disorders among employees of the Family Health Strategy in Santa Cruz do Sul, RS, and to describe sociodemographic and occupational factors associated with these disorders. Methods: This was a cross-sectional, quantitative study, conducted from June to August 2013. In total, 83 employees, who worked at 11 family health units in the city, participated. Participants included doctors, nurses, technicians/nursing assistants, and community health workers. The survey was conducted using a self-administered questionnaire about sociodemographic and occupational issues as well as the matters related to the Self-Reporting Questionnaire. Results: The average age of participants was $37.1 \pm 8.9$ years. The majority of community health workers were female workers who were married, had children, had a per capita income of up to 2 minimum wages, and had completed high school. The general prevalence of common mental health disorders was $19.7 \%$. Mental health disorders were present in all occupational categories, but were more frequent among male nurses (25\%), although this was not statistically significant. Conclusion: This study, in addition to characterizing the population of employees of the Family Health Strategy in Santa Cruz do Sul, RS, in terms of demographic and occupational characteristics, found that psychological distress was present, to varying degrees, in all professional categories evaluated. Given the high prevalence of mental health disorders among these employees, measures are required to ensure the protection and promotion of mental health in this setting.
\end{abstract}

\section{Resumen}

Objetivo: Se ha objetivado describir a los factores sociodemográficos y ocupacionales y evaluar la prevalencia de trastornos mentales comunes en trabajadores de las Estrategias de Salud de la Familia en Santa Cruz do Sul, RS. Métodos: Estudio con abordaje epidemiológico, de corte transversal y cuantitativo, llevado a efecto en el periodo de junio a agosto del 2013. Han sido evaluados 83 trabajadores, entre médicos, enfermeros, técnicos y auxiliares de enfermería y agentes comunitarios de salud pública, que actúan en las 11 unidades de salud de la familia en el municipio. El instrumento de investigación constituyó de un cuestionario auto aplicable, que abarca aspectos sociodemográficos, ocupacionales y cuestiones pertenecientes al Self-Reporting Questionnaire. Resultados: La edad promedia de los participantes fue de $37,1 \pm 8,9$ años, siendo los más, agentes comunitarios de salud, del sexo femenino, casadas, con hijos, ingreso per cápita de hasta dos sueldos mínimos y enseñanza media cumplida. La prevalencia general de trastorno mental común ha sido del $19,7 \%$, estando presente en todos los rangos profesionales y más frecuente entre los enfermeros (25\%), pero sin distinciones estadísticas. Conclusión: Este estudio, además de la caracterización de la población de empleados de Estrategias de Salud de la Familia de Santa Cruz do Sul, RS, en sus características demográficas y ocupacionales, se encontró que la angustia psicológica se encuentra presente, aunque en distintos grados, en todas las categorías profesionales evaluados. Frente a la prevalencia elevada de trastornos mentales, se sugiere providencias con vistas a la prevención y promoción de la salud mental de esos profesionales.
Keywords:

Primary Health Care

Occupational Health

Mental Disorders

Stress, Psychological

Community Health Workers

Palabras clave:

Atención Primaria de Salud

Salud Laboral

Trastornos Mentales

Estrés Psicológico

Agentes Comunitarios de Salud

\section{Introdução}

Estratégia de Saúde da Família (ESF) é uma política do Ministério da Saúde que tem como eixo norteador a reorganização, orientação e o fortalecimento da atenção básica à saúde no Brasil, tendo como prioridade $o$ atendimento integral e continuado dos indivíduos, das famílias e comunidades por meio do trabalho em equipe. Visa à conversão do modelo assistencial tradicional (biomédico) para o modelo de saúde da família. ${ }^{1,2}$

As propostas de atendimento nas ESF ainda são recentes e faz-se necessário que os profissionais estejam preparados e capacitados para enfrentar de modo satisfatório essas mudanças. ${ }^{3}$ Essa exigência, associada ao fato das unidades de saúde da família estarem, muitas vezes, inseridas em ambientes perigosos e insalubres marcados por desigualdades sociais e pobreza, pode favorecer o surgimento de sofrimento psíquico ${ }^{4-7}$ nesses trabalhadores.

Segundo a Organização Mundial da Saúde ${ }^{8}$, saúde mental pode ser definida como um estado de bem-estar no qual o indivíduo desenvolve atributos que lhe permitem enfrentar as tensões normais da vida, estando apto para trabalhar de forma produtiva e contribuir à sociedade na qual está inserido. Assim, esse conceito vai além da simples ausência de transtornos mentais, sendo determinado pela complexa interface que se estabelece entre as características dos indivíduos e seu ambiente de inserção social. ${ }^{8,9}$ 
Atualmente, os distúrbios psiquiátricos podem ser considerados problemas graves de saúde pública. Os transtornos mentais correspondem a $12 \%$ da carga mundial de doenças e a $1 \%$ da mortalidade. ${ }^{8}$ Estima-se que em 2020 serão responsáveis por 15\% de DALYs (Anos de Vida Perdidos por Morte Prematura Ajustados por Incapacidade). ${ }^{8,10}$

Nesse contexto, destacam-se os transtornos mentais comuns (TMC), que se manifestam por meio de quadros clínicos inespecíficos e não psicóticos, tais como insônia, nervosismo, irritabilidade, fadiga, dores de cabeça e esquecimentos. ${ }^{11}$ Apesar de não preencherem todos os critérios necessários para serem classificados como doença mental, de acordo com o Diagnosticand Statistical Manual of Mental Disorders - Fourth Edition (DSM IV) e a Classificação Internacional de Doenças - 10 Revisão (CID-10), são responsáveis por quadros de sofrimento psíquico com significativo comprometimento da qualidade de vida, podendo gerar incapacidades funcionais tão ou mais graves do que aquelas estabelecidas em quadros crônicos. ${ }^{5,12-14}$

Em relação à população de trabalhadores ativos, nota-se que questões relacionadas à precarização do trabalho têm sido apontadas como fatores determinantes na piora das condições de saúde e, consequentemente, na mudança do perfil epidemiológico das doenças relacionadas com a atividade laboral, com destaque para os transtornos mentais. ${ }^{15,16}$ Estima-se que 30\% dos indivíduos que trabalham sejam acometidos por transtornos mentais leves, enquanto que 5\% a 10\% têm transtornos mentais graves. ${ }^{8}$

Entre os trabalhadores da saúde, as prevalências de TMC têm sido estimadas por alguns estudos em diversos ambientes laborais, revelando dados expressivos e preocupantes. ${ }^{17,18} \mathrm{~A}$ exposição desses profissionais a situações de risco biológico, físico e psíquico inerentes à profissão pode estar associada ao desenvolvimento de sofrimento psíquico, com repercussões negativas no desempenho laboral e na própria sociedade..$^{5,7}$

Apesar do importante trabalho desenvolvido no âmbito das ESF no contexto do Sistema Único de Saúde (SUS), poucos estudos se dedicam à avaliação do perfil sociodemográfico e da saúde psíquica desses trabalhadores. Assim, optou-se pela elaboração deste estudo, que tem por objetivo descrever as características sociodemográficas, variáveis ocupacionais e a prevalência de TMC entre médicos, enfermeiros, técnicos/auxiliares de enfermagem e agentes comunitários de saúde nas Estratégias de Saúde da Família em Santa Cruz do Sul, RS.

\section{Métodos}

Trata-se de um estudo com abordagem epidemiológica, de corte transversal e quantitativo. Foram selecionados para participar da pesquisa todos os médicos, enfermeiros, técnicos/auxiliares de enfermagem (TE) e agentes comunitários de saúde (ACS) que compõem as 11 equipes de estratégia de saúde da família do município em estudo, não sendo necessário, portanto, cálculo de tamanho amostral. Excluímos os participantes que, por motivos pessoais, se recusaram a responder o questionário estruturado e que não estiveram presentes no momento da coleta após três tentativas de entrevista agendada previamente.

A coleta de dados ocorreu no período de junho a agosto de 2013 , sendo realizada por entrevistadores submetidos a treinamento para abordagem dos profissionais nas unidades de saúde. Desse modo, os trabalhadores elegíveis para o estudo receberam explicações referentes à pesquisa e, os que concordaram em participar, assinaram o Termo de Consentimento Livre e Esclarecido do estudo. 
O instrumento de pesquisa constituiu-se em um questionário autoaplicável, identificado apenas pela unidade de saúde e composto por três blocos que avaliaram aspectos sociodemográficos (sexo, idade, escolaridade, estado civil, presença de filhos e renda familiar per capita), aspectos ocupacionais (categoria profissional, carga horária semanal, tempo de trabalho na instituição, tipo de contrato de trabalho) e vinte questões pertencentes ao Self-Reporting Questionnaire (SRQ-20). Os questionários foram distribuídos e coletados no mesmo dia, sendo respondidos em um ambiente adequado da própria unidade de saúde.

O SRQ-20, utilizado para avaliar sofrimento psíquico, foi desenvolvido em 1980 por Harding et al., ${ }^{19}$ sendo traduzido e validado para uso no Brasil em 1986 por Mari e Williams. ${ }^{20}$ É composto por vinte questões com respostas do tipo dicotômicas: sim/não, atribuindo-se, respectivamente, valores de 1 e 0 e levando-se em consideração a presença ou não dos sintomas questionados nos últimos 30 dias. A partir das respostas, obtém-se um escore.

No presente estudo, utilizaremos o ponto de corte de seis ou mais respostas positivas para homens, e oito ou mais respostas positivas para mulheres. Indivíduos com score acima desses valores são considerados prováveis casos de TMC que, embora não sejam classificados como portadores de um diagnóstico psiquiátrico formal, podem apresentar sofrimento mental relevante. O teste apresenta sensibilidade de $83 \%$ e especificidade de $80 \%$, com uma taxa de classificação equivocada de $19 \%$ para detecção de transtornos psiquiátricos não psicóticos.

Os dados coletados foram inseridos numa base de dados do programa estatístico Statistic Package for Social Sciences (SPSS) versão 20 e tiveram dupla digitação para diminuição de erros de consistência, sendo analisados a partir da estatística descritiva. Para detectar a existência de associações entre as categorias profissionais e o SRQ-20, utilizou-se o Teste Exato de Fisher, estabelecendo-se um nível de significância de $5 \%$.

O estudo foi aprovado pelo Comitê de Ética em Pesquisa (CEP) da Universidade de Santa Cruz do Sul, RS, sob CAAE número 06706912.3.0000.5343 e pela coordenação da Secretaria de Saúde do município.

\section{Resultados}

Do total da população alvo (89 trabalhadores), um estava em licença-maternidade na época da coleta e 5 se recusaram a participar da pesquisa, totalizando 83 participantes (93,2\%). Para análise de transtorno mental comum pelo SRQ-20, sete participantes $(8,4 \%)$ foram excluídos devido ao fato de não terem preenchido algum item do questionário.

Foram avaliados $48 \mathrm{ACS}$, vinte TE, 8 enfermeiros e 7 médicos. A maioria dos trabalhadores era do sexo feminino (95,1\%), com idade média de $37,1 \pm 8,9$ anos, casados ou com companheiro (a) $(80,2 \%)$, com até dois filhos $(68,7 \%)$ e renda familiar per capita de até dois salários mínimos da época $(R \$ 678,00)$ (75\%). Em relação à escolaridade, possuíam ensino médio completo $(43,9 \%)$ e, dos que possuíam nível superior, 68,7\% referiram curso de pós-graduação completo (Tabela 1).

Entre as variáveis ocupacionais, a maior parcela dos trabalhadores participantes foi constituída por ACS $(57,8 \%)$, com carga horária semanal de 40 horas e dedicação exclusiva $(80,7 \%)$ e com tempo de trabalho na instituição entre 6 meses a 5 anos $(61,4 \%)$. Todos os entrevistados referiram contrato de trabalho regido pela Consolidação das Leis Trabalhistas (CLT), sem ingresso por concurso público (Tabela 2). 
Tabela 1. Distribuição dos trabalhadores das Estratégias Saúde da Família, segundo variáveis sociodemográficas. Santa Cruz do Sul, RS, 2013 ( $n=83$ ).

\begin{tabular}{|c|c|c|}
\hline Variáveis Sociodemográficas & $\mathbf{n}$ & $\%$ \\
\hline \multicolumn{3}{|l|}{ Sexo $(n=82)$} \\
\hline Feminino & 78 & 95,1 \\
\hline Masculino & 4 & 4,9 \\
\hline \multicolumn{3}{|c|}{ Idade (anos completos) $(n=80)-37,1( \pm 8,9)^{*}$} \\
\hline até 30 anos & 25 & 31,3 \\
\hline 31 a 35 anos & 15 & 18,8 \\
\hline 36 a 45 anos & 25 & 31,3 \\
\hline$>45$ anos & 15 & 18,8 \\
\hline \multicolumn{3}{|l|}{ Escolaridade $(n=82)$} \\
\hline Ensino fundamental & 5 & 6,1 \\
\hline Ensino médio & 36 & 43,9 \\
\hline Ensino técnico & 25 & 30,5 \\
\hline Ensino superior & 16 & 19,5 \\
\hline \multicolumn{3}{|l|}{ Estado Civil $(n=81)$} \\
\hline Casado(a) ou com companheiro(a) & 65 & 80,2 \\
\hline Solteiro(a) & 16 & 19,8 \\
\hline \multicolumn{3}{|l|}{ Filhos $(n=83)$} \\
\hline Nenhum & 14 & 16,9 \\
\hline 1 a 2 filhos & 57 & 68,7 \\
\hline Mais de 2 filhos & 12 & 14,5 \\
\hline \multicolumn{3}{|l|}{ Renda familiar per capita $(n=80)$} \\
\hline Até 2 salários mínimos & 60 & 75 \\
\hline Mais de 2 salários mínimos & 20 & 25 \\
\hline
\end{tabular}

Tabela 2. Distribuição dos trabalhadores das Estratégias Saúde da Família, segundo variáveis ocupacionais. Santa Cruz do Sul, RS, 2013 ( $n=83$ ).

\begin{tabular}{|c|c|c|c|}
\hline Variáveis Ocupacionais & & $\mathbf{n}$ & $\%$ \\
\hline \multicolumn{4}{|l|}{ Categoria Profissional } \\
\hline & Agente Comunitário de Saúde & 48 & 57,8 \\
\hline & Técnico de Enfermagem & 20 & 24,1 \\
\hline & Enfermeiro & 8 & 9,6 \\
\hline & Médico & 7 & 8,4 \\
\hline \multicolumn{4}{|l|}{ Carga horária semanal } \\
\hline & 40h/semana (Dedicação Exclusiva) & 67 & 80,7 \\
\hline & Mais de $40 \mathrm{~h} /$ semana & 16 & 19,3 \\
\hline \multicolumn{4}{|c|}{ Tempo de trabalho na Instituição } \\
\hline & 06 meses a 05 anos & 51 & 61,4 \\
\hline & 06 a 09 anos & 20 & 24,1 \\
\hline & $\geq$ de 10 anos & 12 & 14,5 \\
\hline
\end{tabular}


Em relação à saúde mental dos trabalhadores, observa-se que a prevalência geral de TMC, de acordo com o ponto de corte adotado, foi de 19,7\%, observando-se uma variação de 14,3\% (entre os médicos) a $25 \%$ (entre os enfermeiros). Não se encontrou diferença estatística significativa entre a presença de TMC e as categorias avaliadas $(p=0,91)$ (Tabela 3$)$.

Tabela 3. Prevalência de transtorno mental comum entre trabalhadores das Estratégias Saúde da Família, segundo categoria profissional. Santa Cruz do Sul, RS, $2013(n=76)$. **

\begin{tabular}{|c|c|c|c|c|c|c|}
\hline \multirow{3}{*}{ Categoria Profissional } & \multicolumn{6}{|c|}{ Transtorno Mental Comum } \\
\hline & \multirow[b]{2}{*}{$\mathbf{n}$} & \multicolumn{2}{|c|}{ Ausente } & \multicolumn{2}{|c|}{ Presente } & \multirow[t]{2}{*}{$\mathbf{p}^{*}$} \\
\hline & & $\mathbf{n}$ & $\%$ & $\mathbf{n}$ & $\%$ & \\
\hline Agente Comunitário de Saúde & 42 & 33 & 78,6 & 9 & 21,4 & \\
\hline Técnico de Enfermagem & 19 & 16 & 84,2 & 3 & 15,8 & \\
\hline Enfermeiro & 8 & 6 & 75 & 2 & 25 & 0,91 \\
\hline Médico & 7 & 6 & 85,7 & 1 & 14,3 & \\
\hline Total & 76 & 61 & 80,3 & 15 & 19,7 & \\
\hline
\end{tabular}

Todos os casos de TMC ocorreram no sexo feminino $(n=15)$, sendo mais frequentes entre os profissionais com idade entre 36 a 45 anos ( $7 / 15$ ou $46,6 \%$ ), com ensino médio completo ( $8 / 15$ ou $53,3 \%$ ), casados (14/15 ou 93,3\%), com até 2 filhos (12/15 ou $80 \%$ ), renda per capita de até 2 salários mínimos da época (12/15 ou 80\%), com jornada laboral de 40 horas/semana e dedicação exclusiva (12/15 ou 80\%) e com tempo de trabalho na mesma instituição entre 6 a 9 anos (7/15 ou 46,6\%).

As Tabelas 4 e 5 mostram a distribuição dos casos de TMC, entre as categorias profissionais, segundo as variáveis sociodemográficas e ocupacionais.

\section{Discussão}

No município de Santa Cruz do Sul, RS, no momento da coleta dos dados do presente estudo, verificou-se que as 11 ESF apresentavam-se em concordância com o preconizado pelo Ministério da Saúde em relação à modalidade mínima de formação das equipes de saúde da família: um médico generalista ou com formação em saúde da família, um enfermeiro, um ou dois TE e de 4 a 6 ACS. ${ }^{21}$

Observou-se predominância do sexo feminino entre os profissionais das ESF estudadas, independentemente da categoria profissional. Tal situação pode corresponder ao processo de feminização das profissões relacionadas à área da saúde e, consequentemente, dos profissionais das equipes de saúde da família. ${ }^{22,23}$ Fatores como aumento no nível de escolaridade e instrução, em associação com a diminuição das taxas de fecundidade, podem explicar o fenômeno de inserção feminina no mercado de trabalho formal e remunerado. ${ }^{24}$

Outro fator a ser destacado, ainda em relação às mulheres, refere-se à carga de trabalho desenvolvida no âmbito doméstico que, em algumas situações, configura uma dupla ou até tripla jornada. ${ }^{17}$ As tarefas domésticas muitas vezes não são valorizadas e podem representar uma sobrecarga laboral, contribuindo de forma significativa para o adoecimento físico e mental das trabalhadoras. ${ }^{7}$

Os casos de TMC foram mais frequentes entre os profissionais casados e com filhos, ainda que não tenhamos detectado diferenças significativas em comparação com as demais categorias estudadas. 
Moreira IJB, Horta JA, Duro LN, Borges DT, Cristofari AB, Chaves J et al.

Tabela 4. Prevalência de transtorno mental comum entre trabalhadores das Estratégias Saúde da Família por categoria profissional, segundo variáveis sociodemográficas. Santa Cruz do Sul, RS, $2013(n=76){ }^{* *}$

\begin{tabular}{|c|c|c|c|c|c|c|c|c|c|c|}
\hline \multirow{4}{*}{ Variáveis Sociodemográficas } & \multicolumn{10}{|c|}{ Categoria Profissional } \\
\hline & \multicolumn{3}{|c|}{$\begin{array}{l}\text { Agente Comunitário } \\
\text { de Saúde }\end{array}$} & \multicolumn{3}{|c|}{$\begin{array}{l}\text { Técnico/Auxiliar } \\
\text { de Enfermagem }\end{array}$} & \multirow{2}{*}{\multicolumn{2}{|c|}{$\begin{array}{c}\text { Enfermeiro } \\
\text { TMC }\end{array}$}} & \multirow{2}{*}{\multicolumn{2}{|c|}{$\begin{array}{l}\text { Médico } \\
\text { TMC }\end{array}$}} \\
\hline & \multicolumn{2}{|c|}{ TMC } & \multirow{2}{*}{$\mathbf{p}^{*}$} & \multicolumn{2}{|c|}{ TMC } & \multirow{2}{*}{$\mathbf{p}^{*}$} & & & & \\
\hline & $\mathbf{n}$ & $\%$ & & $\mathbf{n}$ & $\%$ & & $\mathbf{n}$ & $\%$ & $\mathbf{n}$ & $\%$ \\
\hline \multicolumn{11}{|l|}{ Sexo } \\
\hline Feminino & 9 & 22,5 & & 3 & 16,7 & & 2 & 25 & 1 & 20 \\
\hline Masculino & 0 & 0 & & 0 & 0 & & 0 & 0 & 0 & 0 \\
\hline \multicolumn{11}{|l|}{ Idade (anos completos) } \\
\hline até 30 anos & 3 & 20 & \multirow{4}{*}{0,6} & 1 & 14,3 & \multirow{4}{*}{0,7} & 0 & 0 & 1 & 50 \\
\hline 31 a 35 anos & 2 & 28,6 & & 0 & 0 & & 0 & 0 & 0 & 0 \\
\hline 36 a 45 anos & 4 & 28,6 & & 1 & 25 & & 2 & 100 & 0 & 0 \\
\hline$>45$ anos & 0 & 0 & & 1 & 16,7 & & 0 & 0 & 0 & 0 \\
\hline \multicolumn{11}{|l|}{ Escolaridade } \\
\hline Ensino fundamental & 0 & 0 & \multirow{4}{*}{0,7} & 0 & 0 & & 0 & 0 & 0 & 0 \\
\hline Ensino médio & 8 & 25,8 & & 0 & 0 & & 0 & 0 & 0 & 0 \\
\hline Ensino técnico & 1 & 20 & & 3 & 15,8 & & 0 & 0 & 0 & 0 \\
\hline Ensino superior & 0 & 0 & & 0 & 0 & & 2 & 25 & 1 & 14,3 \\
\hline \multicolumn{11}{|l|}{ Estado Civil } \\
\hline Casado(a) ou com companheiro(a) & 9 & 25 & & 3 & 18,7 & & 2 & 33,3 & 0 & 0 \\
\hline Solteiro(a) & 0 & 0 & & 0 & 0 & & 0 & 0 & 1 & 50 \\
\hline \multicolumn{11}{|l|}{ Filhos } \\
\hline Nenhum & 1 & 33,3 & & 0 & 0 & & 0 & 0 & 1 & 33,3 \\
\hline 1 a 2 filhos & 8 & 25 & 0,3 & 2 & 15,4 & 0,5 & 2 & 66,7 & 0 & 0 \\
\hline Mais de 2 filhos & 0 & 0 & & 1 & 33,3 & & 0 & 0 & 0 & 0 \\
\hline \multicolumn{11}{|l|}{ Renda familiar per capita } \\
\hline Até 2 salários mínimos & 9 & 23,1 & & 3 & 18,7 & & 0 & 0 & 0 & 0 \\
\hline Mais de 2 salários mínimos & 0 & 0 & & 0 & 0 & & 2 & 28,6 & 1 & 14,3 \\
\hline
\end{tabular}

* Teste exato de Fisher. ** Excluídos sete trabalhadores que não preencheram todos os itens do SRQ-20 para avaliação de transtornos mentais comuns.

Tabela 5. Prevalência de transtorno mental comum entre trabalhadores das Estratégias Saúde da Família por categoria profissional, segundo variáveis ocupacionais. Santa Cruz do Sul, RS, $2013(n=76)$. $^{* \star}$

\begin{tabular}{|c|c|c|c|c|c|c|c|c|c|c|c|}
\hline \multirow{4}{*}{ Variáveis Ocupacionais } & \multicolumn{11}{|c|}{ Categoria Profissional } \\
\hline & \multicolumn{3}{|c|}{ Agente Comunitário de Saúde } & \multirow{2}{*}{\multicolumn{3}{|c|}{$\begin{array}{c}\text { Técnico/Auxiliar de Enfermagem } \\
\text { TMC }\end{array}$}} & \multicolumn{3}{|c|}{ Enfermeiro } & \multirow{2}{*}{\multicolumn{2}{|c|}{$\begin{array}{l}\text { Médico } \\
\text { TMC }\end{array}$}} \\
\hline & \multicolumn{2}{|c|}{ TMC } & \multirow{2}{*}{$\mathbf{p}^{*}$} & & & & \multicolumn{2}{|c|}{ TMC } & \multirow{2}{*}{$\mathbf{p}^{*}$} & & \\
\hline & $\mathbf{n}$ & $\%$ & & $\mathbf{n}$ & $\%$ & $\mathbf{p}^{*}$ & $\mathbf{n}$ & $\%$ & & $\mathbf{n}$ & $\%$ \\
\hline \multicolumn{12}{|l|}{ Carga horária semanal } \\
\hline \multicolumn{12}{|c|}{ Tempo de trabalho na Instituição } \\
\hline 06 meses a 05 anos & 3 & 11,5 & & 1 & 11,1 & & 1 & 20 & & 1 & 16,7 \\
\hline 06 a 09 anos & 5 & 50 & 0,06 & 2 & 33,3 & 0,3 & 0 & 0 & 0,2 & 0 & 0 \\
\hline$\geq$ de 10 anos & 1 & 16,7 & & 0 & 0 & & 1 & 100 & & 0 & 0 \\
\hline
\end{tabular}


A mesma situação foi também encontrada em estudos com populações semelhantes. ${ }^{5,15,22}$ É importante salientar que esse fato demanda uma significativa parcela de afazeres extras com o objetivo de conciliar da melhor forma possível o trabalho e a família. ${ }^{25}$ Assim, os indivíduos ficam mais suscetíveis a insatisfações, piora da qualidade de vida, insegurança nos projetos pessoais e perturbação do equilíbrio emocional com consequentes prejuízos à qualidade da assistência prestada aos pacientes. . $^{52}$

Em relação à faixa etária dos profissionais entrevistados, observou-se que se trata de uma equipe de trabalhadores mais experientes, com capacidade para contribuir de forma produtiva no processo laboral ${ }^{22,25}$ e, por isso, com menor chance de adoecer no trabalho. No presente estudo, os TMC foram observados mais frequentemente nos trabalhadores na faixa etária entre 36 a 45 anos, sem, entretanto, mostrar diferenças estatísticas significativas em comparação com as demais faixas de idade avaliadas. A literatura é bastante controversa em relação à associação de sofrimento psíquico e idade.

O grau de instrução da população estudada é bastante relevante e torna-se importante na medida em que a ESF exige profissionais cada vez mais preparados e qualificados para melhor desempenho de suas atividades laborais. ${ }^{3}$ Notou-se que os ACS da população avaliada possuíam bom nível de instrução (75,6\% referiram ter concluído o ensino médio), o que, por sua vez, pode facilitar o processo de trabalho nas ESF, visto que eles são importantes elos entre a comunidade e os serviços. ${ }^{6}$

Alguns estudos revelam associação entre baixa escolaridade, menor renda e desenvolvimento de TMC. ${ }^{17,26}$ Na presente pesquisa, os casos de sofrimento psíquico foram mais frequentes entre os ACS e TE em relação aos enfermeiros e médicos. Entretanto, esses resultados podem ter sido influenciados pelo fato dos trabalhadores sem instrução superior e menor renda constituírem a maior parte da casuística.

Todos os trabalhadores das equipes de saúde da família de Santa Cruz do Sul, RS, na modalidade mínima preconizada pelo Ministério da Saúde, cumprem jornada laboral de 40 horas/semana na unidade de saúde onde estão alocados. Essa caraterística está em conformidade com o preconizado pela Portaria no 648/GM de 28 de março de 2006, que define a jornada laboral de 40 horas semanais para todos os profissionais nas equipes de saúde da família. ${ }^{27}$

Espera-se que o cumprimento da carga horária integral favoreça o desempenho das atividades das equipes de saúde, além da formação de vínculo entre os membros da equipe e desses com a comunidade atendida. Esses fatores refletem diretamente na qualidade do atendimento prestado, uma vez que o próprio paciente passa a confiar na equipe e, por muitas vezes, toma-a como referência, seja para solucionar problemas de saúde ou de ordem pessoal. ${ }^{28}$

O tipo de contrato de trabalho referido por todos os trabalhadores (vínculo celetista/CLT), embora garanta direitos trabalhistas assegurados pela Constituição Federal de 1988, pode dificultar a permanência do profissional no serviço, gerando instabilidade profissional e favorecendo a rotatividade dos trabalhadores nas equipes, com impacto negativo no atendimento da população $0^{5,24}$ e na saúde física e mental dos profissionais.

Observou-se que a prevalência global de TMC encontrada nesse estudo (19,7\%) é um pouco menor do que a prevalência de TMC na população geral, que varia entre 22,7\% e 35\%. ${ }^{6,29}$ Foi maior do que aquelas encontradas em outros dois estudos que avaliaram trabalhadores da atenção primária à saúde (APS) e que utilizaram a mesma metodologia (SRQ-20): Dilélio et al. ${ }^{30}$ constataram uma prevalência de $16 \%$ entre trabalhadores da APS das regiões Sul e Nordeste do Brasil; para Barbosa et al., ${ }^{5}$ a taxa foi de $16 \%$ entre 
profissionais de nível superior de todas as ESF da cidade de Feira de Santana, BA. Entretanto, foi muito inferior àquela encontrada por Braga et al., ${ }^{15}$ que revearam prevalência de 42,6\% entre trabalhadores da rede básica de saúde de Botucatu, SP.

Em relação aos enfermeiros, a taxa de TMC (25\%) foi superior à encontrada em outros estudos: Barbosa et al. ${ }^{5}$ (15,5\% para enfermeiros da ESF), Kirchhof et al. ${ }^{31}$ (18,7\% para enfermeiros de um hospital do Rio Grande do Sul) e Araújo et al. ${ }^{17}$ (20\% para enfermeiros de um hospital de Salvador). Foi inferior à encontrada por Braga et al. ${ }^{15}$ (48\% para enfermeiros da rede básica de Botucatu).

A porcentagem de ACS da amostra com TMC (21,4\%) foi superior àquela encontrada por Dilélio et al. ${ }^{30}$ (18,4\% ACS da APS das regiões Sul e Nordeste do Brasil) e foi inferior à encontrada por Braga et al. $^{15}$ (50,7\% para ACS da rede básica de Botucatu) e por Silva e Menezes ${ }^{29}$ (43,3\% para ACS de unidades básicas em São Paulo).

Os TE da amostra apresentaram prevalência de 15,8\%, que foi inferior às taxas encontradas na literatura por Araújo et al. ${ }^{17}$ (36,4\% para auxiliares de um hospital de Salvador), e por Pinho e Araújo ${ }^{32}$ (20\% para auxiliares do setor de emergência de um hospital de Feira de Santana).

Em relação aos médicos, a taxa de TMC da amostra (14,3\%) foi semelhante aos estudos conduzidos por Dilélio et al. ${ }^{30}$ e Barbosa et al., ${ }^{5}$ cujas taxas foram, respectivamente, $15 \%$ e 17,4\%, e que avaliaram trabalhadores da APS. Foram inferiores aos estudos de Braga et al. ${ }^{15}$ (33\% para médicos da rede básica de Botucatu).

Embora o presente estudo não tenha evidenciado diferença estatisticamente significativa na associação de TCM entre as categorias avaliadas, nota-se uma prevalência maior entre os enfermeiros. A esses profissionais, além dos cuidados de enfermagem inerentes à sua profissão, são designadas funções de gerenciamento, organização e controle do processo de trabalho nas unidades. Desse modo, os enfermeiros estão expostos a altas demandas no trabalho e, com isso, possuem maior risco de sofrimento psíquico. $^{33}$

O modo como os indivíduos reagem a eventos estressores (presentes no meio ambiente ou na vida pessoal), levando-se em conta o nível de tolerância e adaptação frente a situações estressoras, pode ser uma explicação para a variabilidade na prevalência de TMC entre os profissionais avaliados. ${ }^{6}$

A comparação entre os resultados encontrados com os de outros estudos que utilizaram o instrumento SRQ-20 fica, muitas vezes, prejudicada em razão dos diferentes pontos de corte utilizados pelos autores. Nessa pesquisa, optamos pelo ponto de corte diferente para homens e mulheres, conforme sugerido por Mari e Willians ${ }^{20}$ em seu estudo de validação do método no Brasil e também o mesmo adotado por Dilélio et al. ${ }^{30}$ e Braga et al. ${ }^{15}$ para avaliação de trabalhadores da atenção primária à saúde.

Ressalta-se, entretanto, que os estudos mencionados avaliaram trabalhadores pertencentes a Unidades Básicas de Saúde (UBS), ESF e outros centros de atendimento à saúde, o que pode ter acarretado diferentes resultados, levando-se em conta que as características do processo de trabalho são diferentes em cada um desses locais.

Uma segunda limitação diz respeito à característica dos estudos com delineamento transversal, nos quais se observa que tanto a exposição quanto o desfecho ocorrem no mesmo momento, dificultando, assim, o estabelecimento de uma relação de causalidade entre eles. 
Também deve ser considerada a possibilidade de viés do "trabalhador sadio", visto que os profissionais avaliados, somente pelo fato de estarem no mercado de trabalho, talvez sejam mais "saudáveis" que a população geral. Desse modo, apenas os "sobreviventes" ao desfecho avaliado participam do estudo e podem influenciar os resultados achados no estudo (viés de prevalência).

Por outro lado, as prevalências encontradas de TMC na amostra avaliada foram semelhantes e, em alguns casos, superiores a estudos com populações similares, sugerindo maior probabilidade de sofrimento psíquico entre os grupos de trabalhadores avaliados, mesmo se considerarmos apenas os indivíduos que estavam trabalhando no momento da pesquisa.

O uso de questionários autoaplicáveis também é uma limitação e está suscetível a falhas de preenchimento que, por razões éticas e de preservação da identidade, ocasionam perdas de dados que são irreversíveis. Além disso, medidas de caráter autorreferido estão sujeitas a viéses de memória e suspeição diagnóstica, com repercussões nos resultados finais.

\section{Considerações finais}

O presente estudo, além de caracterizar a população de trabalhadores das ESF de Santa Cruz do Sul, RS, em seus aspectos sociodemográficos e ocupacionais, identificou que o sofrimento psíquico está presente, embora em graus variados, em todas as categorias profissionais avaliadas.

Em função do impacto negativo dos transtornos mentais comuns na qualidade de vida e na saúde em geral, orienta-se que medidas de proteção e promoção à saúde do trabalhador sejam analisadas e providenciadas. Nessa perspectiva, sugere-se que espaços de escuta, reflexões e apoio a esses trabalhadores possam ser planejados.

Para entender de forma mais clara e investigar possíveis diferenças entre as categorias profissionais, se fazem necessários estudos complementares para avaliação das condições de vida, das relações interpessoais entre os membros da equipe, além das características psicossociais do trabalho, visto que isso também pode influenciar na saúde mental dos trabalhadores.

\section{Referências}

1. Brasil. Ministério da Saúde. Programa de Saúde da Família. Brasília: Ministério da Saúde; 1994.

2. Brasil. Ministério da Saúde. Secretaria de Atenção à Saúde. Departamento de Atenção Básica. Política nacional de atenção básica. Brasília: Ministério da Saúde; 2006.

3. Ribeiro SFR, Martins STF. Sofrimento psíquico do trabalhador da saúde da família na organização do trabalho. Psicol Estud. 2011;16(2):241-50. DOI: http://dx.doi.org/10.1590/S1413-73722011000200007

4. Trindade LL, Lautert L. Síndrome de Burnout entre os trabalhadores da Estratégia de Saúde da Família. Rev Esc Enferm USP. 2010;44(2):274-9. DOI: http://dx.doi.org/10.1590/S0080-62342010000200005

5. Barbosa GB, Correia AKS, Oliveira LMM, Santos VC, Ferreira SMS, Martins Júnior DF, et al. Trabalho e saúde mental dos profissionais da Estratégia Saúde da Família em um município do Estado da Bahia, Brasil. Rev Bras Saúde Ocup. 2012;37(126):306-15.

6. Resende MC, Azevedo EGS, Lourenço LR, Faria LS, Alves NF, Farina NP, et al. Saúde mental e ansiedade em agentes comunitários que atuam em saúde da família em Uberlândia (MG, Brasil). Ciênc Saúde Coletiva. 2011;16(4):2115-22. DOI: http://dx.doi.org/10.1590/S1413-81232011000400011 
7. Carreiro GSP, Ferreira Filha MO, Lazarte R, Silva AO, Dias MD. O processo de adoecimento mental do trabalhador da Estratégia Saúde da Família. Rev Eletr Enferm. 2013;15(1):146-55. DOI: http://dx.doi.org/10.5216/ree.v15i1.14084

8. World Health Organization. Investing in mental health. Geneva: World Health Organization; 2003.

9. World Health Organization. Mental Health: New Understanding, New Hope. Geneva: World Health Organization; 2001.

10. Silveira DP, Vieira ALS. Saúde mental e atenção básica em saúde: análise de uma experiência no nível local. Ciênc Saúde Coletiva. 2009;14(1):139-48. DOI: http://dx.doi.org/10.1590/S1413-81232009000100019

11. Goldberg D, Huxley P. Commom mental disorders: a bio-social model. London: Tavistock; 1992.

12. Fonseca MLG, Guimarães MBL, Vasconcelos EM. Sofrimento difuso e transtornos mentais comuns: uma revisão bibliográfica. Rev APS. 2008;11(3):285-94.

13. Maragno L, Goldbaum M, Gianini RJ, Novaes HMD, César CLG. Prevalência de transtornos mentais comuns em populações atendidas pelo Programa Saúde da Família (QUALIS) no Município de São Paulo, Brasil. Cad Saúde Pública. 2006;22(8):1639-48. DOI: http://dx.doi.org/10.1590/S0102-311X2006000800012

14. Lyra GFD, Assis SG, Njaine K, Oliveira RVC, Pires TO. A relação entre professores com sofrimento psíquico e crianças escolares com problemas de comportamento. Ciênc Saúde Coletiva. 2009;14(2):435-44. DOI: http://dx.doi.org/10.1590/S1413-81232009000200012

15. Braga LC, Carvalho LR, Binder MCP. Condições de trabalho e transtornos mentais comuns em trabalhadores da rede básica de saúde de Botucatu (SP). Ciênc Saúde Coletiva. 2010;15(Suppl. 1):1585-96. DOI: http://dx.doi.org/10.1590/S1413-81232010000700070

16. Brasil. Ministério da Saúde. Doenças relacionadas ao trabalho: manual de procedimentos para serviços de saúde. Brasília: Ministério da Saúde; 2001.

17. Araújo TM, Aquino E, Menezes G, Santos CO, Aguiar L. Aspectos psicossociais do trabalho e distúrbios psíquicos entre trabalhadoras de enfermagem. Rev Saúde Pública. 2003;37(4):424-33. DOI: http://dx.doi.org/10.1590/S003489102003000400006

18. Nascimento Sobrinho CL, Carvalho FM, Bonfim TAS, Cirino CAS, Ferreira IS. Condições de trabalho e saúde mental dos médicos de Salvador, Bahia, Brasil. Cad Saúde Pública. 2006;22(1):131-40. DOI: http://dx.doi.org/10.1590/S0102$311 \times 2006000100014$

19. Harding TW, de Arango MV, Baltazar J, Climent CE, Ibrahim HH, Ladrido-Ignacio L, et al. Mental disorders in primary health care: a study of their frequency and diagnosis in four developing countries. Psychol Med. 1980y;10(2):231-41. DOI: http://dx.doi.org/10.1017/S0033291700043993

20. Mari JJ, Williams P. A validity study of a psychiatric screening questionnaire (SRQ-20) in primary care in the city of Sao Paulo. Br J Psychiatry. 1986;148:23-6. DOI: http://dx.doi.org/10.1192/bjp.148.1.23

21. Brasil. Ministério da Saúde. Saúde da Família: uma estratégia para reorientação do modelo assistencial. Brasília: Ministério da Saúde; 1997.

22. Camelo SHH, Angerami ELS. Riscos psicossociais relacionados ao trabalho das equipes de saúde da família: percepções dos profissionais. Rev Enferm UERJ. 2007;15(4):502-7.

23. Pinto ESG, Menezes RMP, Villa TCS. Situação de trabalho dos profissionais da Estratégia Saúde da Família em CearáMirim. Rev Esc Enferm USP. 2010;44(3):657-64. DOI: http://dx.doi.org/10.1590/S0080-62342010000300015

24. Costa SM, Prado MCM, Andrade TN, Araújo EPP, Silva Junior WS, Gomes Filho ZC, et al. Perfil do profissional de nível superior nas equipes da Estratégia Saúde da Família em Montes Claros, Minas Gerais, Brasil. Rev Bras Med Fam Com. 2013;8(27):90-6. DOI: http://dx.doi.org/10.5712/rbmfc8(27)530

25. Camelo SHH, Angerami ELS. Formação de recursos humanos para a Estratégia de Saúde da Família. Ciênc Cuid Saúde. 2008;7(1):45-52. DOI: http://dx.doi.org/10.4025/cienccuidsaude.v7i1.4895

26. Ludermir AB. Associação de transtornos mentais comuns com a informalidade das relações de trabalho. J Bras Psiquiatr. 2005;54(3):198-204. 
27. Brasil. Ministério da Saúde. Portaria no 648 de 28 de março de 2006. Aprova a Política Nacional de Atenção Básica estabelecendo a revisão das diretrizes e normas para a organização da Atenção básica para o Programa Saúde da Família (PSF) e Programa Agentes Comunitários de Saúde (PACS). Diário Oficial da República Federativa do Brasil. Brasília: Ministério da Saúde; 2006.

28. Oliveira EM, Spiri WC. Programa Saúde da Família: a experiência de equipe multiprofissional. Rev Saúde Pública. 2006;40(4):727-33. DOI: http://dx.doi.org/10.1590/S0034-89102006000500025

29. Silva ATC, Menezes PR. Esgotamento profissional e transtornos mentais comuns em agentes comunitários de saúde. Rev Saúde Pública. 2008;42(5):921-9. DOI: http://dx.doi.org/10.1590/S0034-89102008000500019

30. Dilélio AS, Facchini LA, Tomasi E, Silva SM, Thumé E, Piccini RX, et al. Prevalência de transtornos psiquiátricos menores em trabalhadores da atenção primária à saúde das regiões Sul e Nordeste do Brasil. Cad Saúde Pública. 2012;28(3):50314. DOI:http://dx.doi.org/10.1590/S0102-311X2012000300011

31. Kirchhof ALC, Magnago TSBS, Camponogara S, Griep RH, Tavares JP, Prestes FC, et al. Condições de trabalho e características sócio-demográficas relacionadas à presença de distúrbios psíquicos menores em trabalhadores de enfermagem. Texto Contexto Enferm. 2009;18(2):215-23. DOI: http://dx.doi.org/10.1590/S0104-07072009000200003

32. Pinho SP, Araújo TM. Trabalho de enfermagem em uma unidade de emergência hospitalar e transtornos mentais. Rev Enferm UERJ. 2007;15(3):329-36.

33. Camelo SHH, Angerami ELS. Sintomas de estresse nos trabalhadores atuantes em cinco núcleos de saúde da família. Rev Latino-Am Enfermagem. 2004;12(1):14-21 DOI: http://dx.doi.org/10.1590/S0104-11692004000100003 